九州大学学術情報リポジトリ

Kyushu University Institutional Repository

On the maximal number of exceptional values of Gauss maps for various classes of surfaces

川上，裕

九州大学大学院数理学府

http://hdl. hand le. net/2324/21978

出版情報: Mathematische Zeitschrift. 274 (3/4)，pp.1249-1260，2012-12. Springer-Verlag バージョン :

権利関係: (C) Springer-Verlag Berlin Heidelberg 2012 


\section{Preprint Series}

Kyushu University

The Global COE Program

Math-for-Industry Education \& Research Hub

\section{On the maximal number of exceptional values of Gauss maps for various classes of surfaces}

\section{Yu Kawakami}

\section{2012-5}

( Received May 22, 2012 )

Faculty of Mathematics

Kyushu University

Fukuoka, JAPAN 


\title{
ON THE MAXIMAL NUMBER OF EXCEPTIONAL VALUES OF GAUSS MAPS FOR VARIOUS CLASSES OF SURFACES
}

\author{
YU KAWAKAMI
}

\begin{abstract}
The main goal of this paper is to reveal the geometric meaning of the maximal number of exceptional values of Gauss maps for several classes of immersed surfaces in space forms, for example, complete minimal surfaces in the Euclidean three-space, weakly complete improper affine spheres in the affine three-space and weakly complete flat surfaces in the hyperbolic three-space. For this purpose, we give an effective curvature bound for a specified conformal metric on an open Riemann surface.
\end{abstract}

\section{INTRODUCTION}

The geometric nature of value distribution theory of complex analytic mappings is wellknown. One of the most elegant results of the theory is the geometric meaning of the precise maximum "2" for the number of exceptional values of nonconstant meromorphic functions on the complex plane C. In fact, Ahlfors [1] and Chern [5] showed that the maximal number of exceptional values of nonconstant holomorphic maps from $\mathbf{C}$ to a closed Riemann surface coincides with the Euler number of the closed Riemann surface by using Nevanlinna theory (see also [21], [29] and [33]). In particular, for nonconstant meromorphic functions on $\mathbf{C}$, the geometric meaning of the maximal number " 2 " of exceptional values is the Euler number of the Riemann sphere.

On the other hand, global properties of the Gauss map of complete minimal surfaces in the Euclidean three-space $\mathbf{R}^{3}$ are closely related to value-distribution-theoretic properties of meromorphic functions on C. In particular, Fujimoto [11] proved that the precise maximum for the number of exceptional values of the Gauss map of a nonflat complete minimal surface in $\mathbf{R}^{3}$ is " 4 ", and Osserman [30] showed that the Gauss map of a nonflat algebraic minimal surface can omit at most 3 values (by an algebraic minimal surface, we mean a complete minimal surface with finite total curvature). Recently, the author, Kobayashi and Miyaoka [18] gave an effective upper bound for the number of exceptional values of the Gauss map for a special class of complete minimal surfaces that includes algebraic minimal surfaces (this class is called the pseudo-algebraic minimal surfaces). This also provided a geometric meaning for the Fujimoto and Osserman results for this

2010 Mathematics Subject Classification. Primary 30D35, 53C42 ; Secondary 30F45, 53A10, 53A15.

Key words and phrases. Gauss map, exceptional value, conformal metric, minimal surface, front, Bernstein type theorem.

Partly supported by the Grant-in-Aid for Young Scientists (B) No. 24740044, Japan Society for the Promotion of Science. 
class, because the upper bound is described in terms of geometric invariants. However, from [18] it was still not possible to understand the geometric meaning for general class.

The author also investigated value-distribution-theoretic properties of Gauss maps for several classes of surfaces which may admit singularities. For instance, by refining the Fujimoto analytic argument, the author and Nakajo [19] showed that the maximal number of exceptional values of the Lagrangian Gauss map of weakly complete improper affine fronts in the affine three-space $\mathbf{R}^{3}$ is " 3 ". As an application of this result, a simple proof of the parametric affine Bernstein theorem for improper affine spheres in $\mathbf{R}^{3}$ was provided. Moreover, the authors $[17,19]$ proved similar results for flat fronts in the hyperbolic three-space $\mathbf{H}^{3}$.

The aim of this paper is to reveal the geometric meaning of the precise maximum for the number of exceptional values of Gauss maps for these classes of surfaces. The paper is organized as follows: In Section 1, we give a curvature bound for the conformal metric $d s^{2}=\left(1+|g|^{2}\right)^{m}|\omega|^{2}$ on an open Riemann surface $\Sigma$, where $\omega$ is a holomorphic 1 -form and $g$ is a meromorphic function on $\Sigma$ (Theorem 1.1). The proof is given in Section 2 . As a corollary of this theorem, we prove that the precise maximum for the number of exceptional values of the nonconstant meromorphic function $g$ on $\Sigma$ with the complete conformal metric $d s^{2}$ is " $m+2$ " (Corollary 1.2 and Proposition 1.4). We note that the geometric meaning of the " 2 " in " $m+2$ " is the Euler number of the Riemann sphere (Remark 1.3). In Section 3, we give some applications of the main results. In particular, we give the geometric meaning of the maximal number of exceptional values of Gauss maps for several classes of immersed surfaces in space forms. For instance, the induced metric from $\mathbf{R}^{3}$ on complete minimal surfaces is $d s^{2}=\left(1+|g|^{2}\right)^{2}|\omega|^{2}$ (i.e. $m=2$ ), thereby the maximal number of exceptional values of the Gauss map $g$ of nonflat complete minimal surfaces in $\mathbf{R}^{3}$ is " $4(=2+2)$ ". On the other hand, for the Lagrangian Gauss map $\nu$ of weakly complete improper affine fronts, since $\nu$ is meromorphic, $d G$ is holomorphic and the complete metric is $d \tau^{2}=2\left(1+|\nu|^{2}\right)|d G|^{2}$ (i.e. $m=1$ ), the maximal number of exceptional values of the Lagrangian Gauss map of weakly complete improper affine fronts in $\mathbf{R}^{3}$ is " $3(=1+2)$ ".

Finally, the author would like to thank Professors Junjiro Noguchi, Wayne Rossman, Masaaki Umehara and Kotaro Yamada for their useful advice. In addition, the author would like to express his thanks to Professors Ryoichi Kobayashi, Masatoshi Kokubu, Miyuki Koiso and Reiko Miyaoka for their encouragement of this study.

\section{Main Results}

Now we state the main theorem of this paper.

THEOREM 1.1. Let $\Sigma$ be an open Riemann surface with the conformal metric

$$
d s^{2}=\left(1+|g|^{2}\right)^{m}|\omega|^{2},
$$


where $\omega$ is a holomorphic 1-form, $g$ is a meromorphic function on $\Sigma$, and $m \in \mathbf{N}$. Suppose that $g$ omits $q \geq m+3$ distinct values. Then there exists a positive constant $C$, depending on $m$ and the set of exceptional values, but not the surface $\Sigma$, such that for all $p \in \Sigma$ we have

$$
\left|K_{d s^{2}}(p)\right|^{1 / 2} \leq \frac{C}{d(p)},
$$

where $K_{d s^{2}}(p)$ is the Gaussian curvature of the metric $d s^{2}$ at $p$ and $d(p)$ is the geodesic distance from $p$ to the boundary of $\Sigma$, that is, the infimum of the lengths of the divergent curves in $\Sigma$ emanating from $p$.

As a corollary of Theorem 1.1, we give the following Picard-type theorem for the meromorphic function $g$ on $\Sigma$ with the complete conformal metric $d s^{2}=\left(1+|g|^{2}\right)^{m}|\omega|^{2}$.

COROLlary 1.2. Let $\Sigma$ be an open Riemann surface with the conformal metric given by (1). If the metric $d s^{2}$ is complete and the meromorphic function $g$ is nonconstant, then $g$ can omit at most $m+2$ distinct values.

Proof. By way of contradiction, assume that $g$ omits $m+3$ distinct values. If $d s^{2}$ is complete, then we may set $d(p)=\infty$ for all $p \in \Sigma$. By virtue of Theorem $1.1, K_{d s^{2}} \equiv 0$ on $\Sigma$. On the other hand, the Gaussian curvature of the metric $d s^{2}$ is given by

$$
K_{d s^{2}}=-\frac{2 m\left|g_{z}^{\prime}\right|^{2}}{\left(1+|g|^{2}\right)^{m+2}\left|\hat{\omega}_{z}\right|^{2}},
$$

where $\omega=\hat{\omega}_{z} d z$ and $g_{z}^{\prime}=d g / d z$. Thus $K_{d s^{2}} \equiv 0$ if and only if $g$ is constant. This contradicts the assumption that $g$ is nonconstant.

REMARK 1.3. The geometric meaning of the "2" in " $m+2$ " is the Euler number of the Riemann sphere. Indeed, if $m=0$ then the metric $d s^{2}=\left(1+|g|^{2}\right)^{0}|\omega|^{2}=|\omega|^{2}$ is flat and complete on $\Sigma$. We thus may assume that $g$ is a meromorphic function on $\mathbf{C}$ because $g$ is replaced by $g \circ \pi$, where $\pi: \mathbf{C} \rightarrow \Sigma$ is a holomorphic universal covering map. On the other hand, Ahlfors [1] and Chern [5] showed that the best possible upper bound "2" of the number of exceptional values of nonconstant meromorphic functions on $\mathbf{C}$ coincides with the Euler number of the Riemann sphere. Hence we get the conclusion.

Corollary 1.2 is optimal because there exist the following examples.

Proposition 1.4. Let $\Sigma$ be either the complex plane punctured at $q-1$ distinct points $\alpha_{1}, \cdots, \alpha_{q-1}$ or the universal cover of that punctured plane. We set

$$
\omega=\frac{d z}{\prod_{i=1}^{q-1}\left(z-\alpha_{i}\right)}, \quad g=z .
$$

Then $g$ omits $q$ distinct values and the metric $d s^{2}=\left(1+|g|^{2}\right)^{m}|\omega|^{2}$ is complete if and only if $q \leq m+2$. In particular, there exist examples whose metric $d s^{2}$ is complete and $g$ omits $m+2$ distinct values. 
Proof. We can easily show that $g$ omits the $q$ distinct values $\alpha_{1}, \cdots, \alpha_{q-1}$ and $\infty$ on $\Sigma$. A divergent curve $\Gamma$ in $\Sigma$ must tend to one of the points $\alpha_{1}, \cdots, \alpha_{q-1}$ or $\infty$. Thus we have

$$
\int_{\Gamma} d s=\int_{\Gamma}\left(1+|g|^{2}\right)^{m / 2}|\omega|=\int_{\Gamma} \frac{\left(1+|z|^{2}\right)^{m / 2}}{\prod_{i=1}^{q-1}\left|z-\alpha_{i}\right|}|d z|=\infty,
$$

when $q \leq m+2$.

\section{Proof of the MaIN Theorem}

We first recall the notion of chordal distance between two distinct values in the Riemann sphere $\mathbf{C} \cup\{\infty\}$. For two distinct values $\alpha, \beta \in \mathbf{C} \cup\{\infty\}$, we set

$$
|\alpha, \beta|:=\frac{|\alpha-\beta|}{\sqrt{1+|\alpha|^{2}} \sqrt{1+|\beta|^{2}}}
$$

if $\alpha \neq \infty$ and $\beta \neq \infty$, and $|\alpha, \infty|=|\infty, \alpha|:=1 / \sqrt{1+|\alpha|^{2}}$. We note that, if we take $v_{1}, v_{2} \in \mathbf{S}^{2}$ with $\alpha=\varpi\left(v_{1}\right)$ and $\beta=\varpi\left(v_{2}\right)$, we have that $|\alpha, \beta|$ is a half of the chordal distance between $v_{1}$ and $v_{2}$, where $\varpi$ denotes the stereographic projection of the 2 -sphere $\mathbf{S}^{2}$ onto $\mathbf{C} \cup\{\infty\}$.

Before proceeding to the proof of Theorem 1.1, we recall two function-theoretical lemmas.

LEMMA 2.1. [13, (8.12) on page 136] Let $g$ be a nonconstant meromorphic function on $\Delta_{R}=\{z \in \mathbf{C} ;|z|<R\}(0<R \leq+\infty)$ which omits $q$ values $\alpha_{1}, \ldots, \alpha_{q}$. If $q>2$, then for each positive $\eta$ with $\eta<(q-2) / q$, then there exists a positive constant $C^{\prime}$, depending on $m, q$ and $L:=\min _{i<j}\left|\alpha_{i}, \alpha_{j}\right|$, such that

$$
\frac{\left|g_{z}^{\prime}\right|}{\left(1+|g|^{2}\right) \prod_{j=1}^{q}\left|g, \alpha_{j}\right|^{1-\eta}} \leq C^{\prime} \frac{R}{R^{2}-|z|^{2}} .
$$

LEMma 2.2. [12, Lemma 1.6.7] Let $d \sigma^{2}$ be a conformal flat metric on an open Riemann surface $\Sigma$. Then, for each point $p \in \Sigma$, there exists a local diffeomorphism $\Phi$ of a disk $\Delta_{R}=\{z \in \mathbf{C} ;|z|<R\}(0<R \leq+\infty)$ onto an open neighborhood of $p$ with $\Phi(0)=p$ such that $\Phi$ is a local isometry, that is, the pull-back $\Phi^{*}\left(d \sigma^{2}\right)$ is equal to the standard Euclidean metric $d s_{E u c}^{2}$ on $\Delta_{R}$ and, for a point $a_{0}$ with $\left|a_{0}\right|=1$, the $\Phi$-image $\Gamma_{a_{0}}$ of the curve $L_{a_{0}}=\left\{w:=a_{0} s ; 0<s<R\right\}$ is divergent in $\Sigma$.

Proof of Theorem 1.1. We may assume that $\alpha_{q}=\infty$. We choose a positive number $\eta$ with

$$
\frac{q-2(m+1)}{q}<\eta<\frac{q-(m+2)}{q}
$$

and set $\lambda:=m /(q-2-q \eta)$. Since $q \geq m+3$, then $0<\lambda<1$ holds.

Now we define a new metric

$$
d \sigma^{2}=\left|\hat{\omega}_{z}\right|^{2 /(1-\lambda)}\left(\frac{1}{\left|g_{z}^{\prime}\right|} \prod_{j=1}^{q-1}\left(\frac{\left|g-\alpha_{j}\right|}{\sqrt{1+\left|\alpha_{j}\right|^{2}}}\right)^{1-\eta}\right)^{2 \lambda /(1-\lambda)}|d z|^{2}
$$


on the set $\Gamma^{\prime}:=\left\{p \in \Sigma ; g_{z}^{\prime}(p) \neq 0\right\}$, where $\omega=\hat{\omega}_{z} d z$ and $g_{z}^{\prime}=d g / d z$ with respect to the local complex coordinate $z$. Take a point $p \in \Sigma^{\prime}$. Since the metric $d \sigma^{2}$ is flat, by Lemma 2.2 , there exists a local isometry $\Phi$ satisfying $\Phi(0)=p$ from a disk $\Delta_{R}=\{z \in \mathbf{C} ;|z|<R\}$ $(0<R \leq+\infty)$ with the standard Euclidean metric $d s_{E u c}^{2}$ onto an open neighborhood of $p \in \Sigma^{\prime}$ with the metric $d \sigma^{2}$, such that, for a point $a_{0}$ with $\left|a_{0}\right|=1$, the $\Phi$-image $\Gamma_{a_{0}}$ of the curve $L_{a_{0}}=\left\{w:=a_{0} s ; 0<s<R\right\}$ is divergent in $\Sigma^{\prime}$. For brevity, we denote the function $g \circ \Phi$ on $\Delta_{R}$ by $g$ in the following. By Lemma 2.1, we get

$$
R \leq C^{\prime} \frac{1+|g(0)|^{2}}{\left|g^{\prime}(0)\right|} \prod_{j=1}^{q}\left|g(0), \alpha_{j}\right|^{1-\eta}<+\infty
$$

Hence

$$
L_{d \sigma}\left(\Gamma_{a_{0}}\right)=\int_{\Gamma_{a_{0}}} d \sigma=R<+\infty
$$

where $L_{d \sigma}\left(\Gamma_{a_{0}}\right)$ denotes the length of $\Gamma_{a_{0}}$ with respect to the metric $d \sigma^{2}$. We assume that the $\Phi$-image $\Gamma_{a_{0}}$ tends to a point $p_{0} \in \Sigma \backslash \Sigma^{\prime}$ as $s \rightarrow R$. Taking a local complex coordinate $\zeta$ in a neighborhood of $p_{0}$ with $\zeta\left(p_{0}\right)=0$, we can write

$$
d \sigma^{2}=|\zeta|^{-2 \lambda /(1-\lambda)} w|d \zeta|^{2}
$$

for some positive smooth function $w$. Since $\lambda /(1-\lambda)>1$, we have

$$
R=\int_{\Gamma_{a_{0}}} d \sigma \geq \widetilde{C} \int_{\Gamma_{a_{0}}} \frac{|d \zeta|}{|\zeta|^{\lambda /(1-\lambda)}}=+\infty
$$

which contradicts (6). Thus $\Gamma_{a_{0}}$ diverges outside any compact subset of $\Sigma$ as $s \rightarrow R$.

Since $d \sigma^{2}=|d z|^{2}$, we obtain by (5) that

$$
\left|\hat{\omega}_{z}\right|=\left(\left|g_{z}^{\prime}\right| \prod_{j=1}^{q-1}\left(\frac{\sqrt{1+\left|\alpha_{j}\right|^{2}}}{\left|g-\alpha_{j}\right|}\right)^{1-\eta}\right)^{\lambda}
$$

By Lemma 2.1, we have

$$
\begin{aligned}
\Phi^{*} d s & =\left|\hat{\omega}_{z}\right|\left(1+|g|^{2}\right)^{m / 2}|d z| \\
& =\left(\left|g_{z}^{\prime}\right|\left(1+|g|^{2}\right)^{m / 2 \lambda} \prod_{j=1}^{q-1}\left(\frac{\sqrt{1+\left|\alpha_{j}\right|^{2}}}{\left|g-\alpha_{j}\right|}\right)^{1-\eta}\right)^{\lambda}|d z| \\
& =\left(\frac{\left|g_{z}^{\prime}\right|}{\left(1+|g|^{2}\right) \prod_{j=1}^{q}\left|g, \alpha_{j}\right|^{1-\eta}}\right)^{\lambda}|d z| \\
& \leq\left(C^{\prime}\right)^{\lambda}\left(\frac{R}{R^{2}-|z|^{2}}\right)^{\lambda}|d z| .
\end{aligned}
$$

Thus we have

$$
d(p) \leq \int_{\Gamma_{a_{0}}} d s=\int_{L_{a_{0}}} \Phi^{*} d s \leq\left(C^{\prime}\right)^{\lambda} \int_{L_{a_{0}}}\left(\frac{R}{R^{2}-|z|^{2}}\right)^{\lambda}|d z| \leq\left(C^{\prime}\right)^{\lambda} \frac{R^{1-\lambda}}{1-\lambda}(<+\infty)
$$


because $0<\lambda<1$. Moreover, by (6), we get that

$$
d(p) \leq \frac{C^{\prime}}{1-\lambda}\left(\frac{1+|g(0)|^{2}}{\left|g^{\prime}(0)\right|} \prod_{j=1}^{q}\left|g(0), \alpha_{j}\right|^{1-\eta}\right)^{1-\lambda} .
$$

On the other hand, the Gaussian curvature $K_{d s^{2}}$ of the metric $d s^{2}=\left(1+|g|^{2}\right)^{m}|\omega|^{2}$ is given by

$$
K_{d s^{2}}=-\frac{2 m\left|g_{z}^{\prime}\right|^{2}}{\left(1+|g|^{2}\right)^{m+2}\left|\hat{\omega}_{z}\right|^{2}} .
$$

Thus, by (7), we also get that

$$
\left|K_{d s^{2}}\right|^{1 / 2}=\sqrt{2 m}\left(\frac{\left|g_{z}^{\prime}\right|}{1+|g|^{2}} \prod_{j=1}^{q}\left|g-\alpha_{j}\right|^{1-\eta}\right)^{1-\lambda} .
$$

Since $\left|g, \alpha_{j}\right| \leq 1$ for each $j$, we obtain that

$$
\left|K_{d s^{2}}\right|^{1 / 2} d(p) \leq \frac{\sqrt{2 m} C^{\prime}}{1-\lambda}=: C .
$$

By the definitions of $C^{\prime}$ and $\lambda$, we see that $C$ is positive and depends on $m, q$ and $L:=\min _{i<j}\left|\alpha_{i}, \alpha_{j}\right|$.

\section{Applications}

In this section, we give several applications of our main results.

3.1. Gauss map of minimal surfaces in the Euclidean 3-space. We briefly recall some basic facts of minimal surfaces in $\mathbf{R}^{3}$. Details can be found, for example, in [12] and [31]. Let $X=\left(x^{1}, x^{2}, x^{3}\right): \Sigma \rightarrow \mathbf{R}^{3}$ be an oriented minimal surface in $\mathbf{R}^{3}$. By associating a local complex coordinate $z=u+\sqrt{-1} v$ with each positive isothermal coordinate system $(u, v), \Sigma$ is considered as a Riemann surface whose conformal metric is the induced metric $d s^{2}$ from $\mathbf{R}^{3}$. Then

$$
\triangle_{d s^{2}} X=0
$$

holds, that is, each coordinate function $x^{i}$ is harmonic. With respect to the local complex coordinate $z=u+\sqrt{-1} v$ of the surface, (11) is given by

$$
\bar{\partial} \partial X=0,
$$

where $\partial=(\partial / \partial u-\sqrt{-1} \partial / \partial v) / 2$ and $\bar{\partial}=(\partial / \partial u+\sqrt{-1} \partial / \partial v) / 2$. Hence each $\phi_{i}:=\partial x^{i} d z$ $(i=1,2,3)$ is a holomorphic 1 -form on $\Sigma$. If we set

$$
\omega=\phi_{1}-\sqrt{-1} \phi_{2}, \quad g=\frac{\phi_{3}}{\phi_{1}-\sqrt{-1} \phi_{2}},
$$


then $\omega$ is a holomorphic 1-form and $g$ is a meromorphic function on $\Sigma$. Moreover, the function $g$ coincides with the composition of the Gauss map and the stereographic projection from $\mathbf{S}^{2}$ onto $\mathbf{C} \cup\{\infty\}$, and the induced metric $d s^{2}$ is given by

$$
d s^{2}=\left(1+|g|^{2}\right)^{2}|\omega|^{2} .
$$

Applying Theorem 1.1 to the metric $d s^{2}$, we can show the Fujimoto theorem for the Gauss map of minimal surfaces in $\mathbf{R}^{3}$.

Theorem 3.1. [11, Theorem I and Corollary 3.4] Let $X: \Sigma \rightarrow \mathbf{R}^{3}$ be an oriented minimal surface whose Gauss map $g: \Sigma \rightarrow \mathbf{C} \cup\{\infty\}$ omits more than $4(=2+2)$ distinct values. Then there exists a positive constant $C$ depending on the set of exceptional values, but not the surface, such that for all $p \in \Sigma$ the inequality (2) holds. In particular, the Gauss map of a nonflat complete minimal surface in $\mathbf{R}^{3}$ can omit at most $4(=2+2)$ values.

\subsection{Lorentzian Gauss map of maxfaces in the Lorentz-Minkowski 3-space.} Maximal surfaces in the Lorentz-Minkowski 3-space $\mathbf{R}_{1}^{3}$ are closely related to minimal surfaces in $\mathbf{R}^{3}$. In this subsection we treat maximal surfaces with some admissible singularities, called maxfaces, as introduced by Umehara and Yamada [36]. We remark that maxfaces, non-branched generalized maximal surfaces in the sense of [9] and non-branched generalized maximal maps in the sense of [15] are all the same class of maximal surfaces. The Lorentz-Minkowski 3-space $\mathbf{R}_{1}^{3}$ is the affine 3-space $\mathbf{R}^{3}$ with the inner product

$$
\langle,\rangle=-\left(d x^{1}\right)^{2}+\left(d x^{2}\right)^{2}+\left(d x^{3}\right)^{2},
$$

where $\left(x^{1}, x^{2}, x^{3}\right)$ is the canonical coordinate system of $\mathbf{R}^{3}$. We consider a fibration

$$
p_{L}: \mathbf{C}^{3} \ni\left(\zeta^{1}, \zeta^{2}, \zeta^{3}\right) \mapsto \operatorname{Re}\left(-\sqrt{-1} \zeta^{3}, \zeta^{1}, \zeta^{2}\right) \in \mathbf{R}_{1}^{3}
$$

The projection of null holomorphic immersions into $\mathbf{R}_{1}^{3}$ by $p_{L}$ gives maxfaces. Here, a holomorphic map $F=\left(F_{1}, F_{2}, F_{3}\right): \Sigma \rightarrow \mathbf{C}^{3}$ is called null if $\left\{\left(F_{1}\right)_{z}^{\prime}\right\}^{2}+\left\{\left(F_{2}\right)_{z}^{\prime}\right\}^{2}+\left\{\left(F_{3}\right)_{z}^{\prime}\right\}^{2}$ vanishes identically, where ${ }^{\prime}=d / d z$ denotes the derivative with respect to a local complex coordinate $z$ of $\Sigma$. For maxfaces, an analogue of the Enneper-Weierstrass representation formula is known (see also [20]).

Theorem 3.2. [36, Theorem 2.6] Let $\Sigma$ be a Riemann surface and $(g, \omega)$ a pair consisting of a meromorphic function and a holomorphic 1-form on $\Sigma$ such that

$$
d \sigma^{2}:=\left(1+|g|^{2}\right)^{2}|\omega|^{2}
$$

gives a (positive definite) Riemannian metric on $\Sigma$, and $|g|$ is not identically 1. Assume that

$$
\operatorname{Re} \int_{\gamma}\left(-2 g, 1+g^{2}, \sqrt{-1}\left(1-g^{2}\right)\right) \omega=0
$$


for all loops $\gamma$ in $\Sigma$. Then

$$
f=\operatorname{Re} \int_{z_{0}}^{z}\left(-2 g, 1+g^{2}, \sqrt{-1}\left(1-g^{2}\right)\right) \omega
$$

is well-defined on $\Sigma$ and gives a maxface in $\mathbf{R}_{1}^{3}$, where $z_{0} \in \Sigma$ is a base point. Moreover, all maxfaces are obtained in this manner. The induced metric $d s^{2}:=f^{*}\langle$,$\rangle is given by$

$$
d s^{2}=\left(1-|g|^{2}\right)^{2}|\omega|^{2},
$$

and the point $p \in \Sigma$ is a singular point of $f$ if and only if $|g(p)|=1$.

We call $g$ the Lorentzian Gauss map of $f$. If $f$ has no singularities, then $g$ coincides with the composition of the Gauss map (i.e., (Lorentzian) unit normal vector) $n: \Sigma \rightarrow \mathbf{H}_{ \pm}^{2}$ into the upper or lower connected component of the two-sheet hyperboloid $\mathbf{H}_{ \pm}^{2}=\mathbf{H}_{+}^{2} \cup \mathbf{H}_{-}^{2}$ in $\mathbf{R}_{1}^{3}$, where

$$
\begin{aligned}
& \mathbf{H}_{+}^{2}:=\left\{n=\left(n^{1}, n^{2}, n^{3}\right) \in \mathbf{R}_{1}^{3} ;\langle n, n\rangle=-1, n^{1}>0\right\} \\
& \mathbf{H}_{-}^{2}:=\left\{n=\left(n^{1}, n^{2}, n^{3}\right) \in \mathbf{R}_{1}^{3} ;\langle n, n\rangle=-1, n^{1}<0\right\}
\end{aligned}
$$

and the stereographic projection from the north pole $(0,0,1)$ of the hyperboloid onto the Riemann sphere $\mathbf{C} \cup\{\infty\}$ (see [36, Section 1]). A maxface is said to be weakly complete if the metric $d \sigma^{2}$ as in (15) is complete. We note that $(1 / 2) d \sigma^{2}$ coincides with the pull-back of the standard metric on $\mathbf{C}^{3}$ by the null holomorphic immersion of $f$ (see [36, Section $2])$.

Applying Theorem 1.1 to the metric $d \sigma^{2}$, we can get the following theorem.

Theorem 3.3. Let $f: \Sigma \rightarrow \mathbf{R}_{1}^{3}$ be a maxface whose Lorentzian Gauss map $g: \Sigma \rightarrow$ $\mathbf{C} \cup\{\infty\}$ omits more than $4(=2+2)$ distinct values. Then there exists a positive constant $C$ depending on the set of exceptional values, but not $\Sigma$, such that for all $p \in \Sigma$ we have

$$
\left|K_{d \sigma^{2}}(p)\right|^{1 / 2} \leq \frac{C}{d(p)},
$$

where $K_{d \sigma^{2}}(p)$ is the Gaussian curvature of the metric $d \sigma^{2}$ at $p$ and $d(p)$ is the geodesic distance from $p$ to the boundary of $\Sigma$. In particular, the Lorentzian Gauss map of a nonflat weakly complete maxface in $\mathbf{R}_{1}^{3}$ can omit at most $4(=2+2)$ values.

As a corollary of this result, we give a simple new proof of the Calabi-Bernstein theorem ([4], [7]) for maximal space-like surfaces in $\mathbf{R}_{1}^{3}$ from the viewpoint of value-distributiontheoretic properties of the Lorentzian Gauss map. We remark that Alías and Palmer [2], Estudillo and Romero [8, 9, 10], Osamu Kobayashi [20], Romero [32] and Umehara and Yamada [36] have approached this theorem from other viewpoints.

COROLlary 3.4. Any complete maximal space-like surface in $\mathbf{R}_{1}^{3}$ must be a plane. 
Proof. Since a maximal space-like surface has no singularities, the complement of the image of $g$ contains at least the set $\{|g|=1\} \subset \mathbf{C} \cup\{\infty\}$. On the other hand, we obtain

$$
d s^{2}=\left(1-|g|^{2}\right)^{2}|\omega|^{2} \leq\left(1+|g|^{2}\right)^{2}|\omega|^{2}=d \sigma^{2} .
$$

Thus if $d s^{2}$ is complete, then $d \sigma^{2}$ is also complete. By Theorem 3.3, its Lorentzian Gauss map is constant, that is, it is a plane.

3.3. Lagrangian Gauss map of improper affine fronts in the affine 3-space. Improper affine spheres in the affine 3 -space $\mathbf{R}^{3}$ also have similar properties to minimal surfaces in the Euclidean 3-space. Recently, Martínez [27] discovered the correspondence between improper affine spheres and smooth special Lagrangian immersions in the complex 2-space $\mathbf{C}^{2}$ and introduced the notion of improper affine fronts, that is, a class of (locally strongly convex) improper affine spheres with some admissible singularities in $\mathbf{R}^{3}$. We note that this class is called "improper affine maps" in [27], but we call this class "improper affine fronts" because Nakajo [28] and Umehara and Yamada [37, 38] showed that all improper affine maps are wave fronts in $\mathbf{R}^{3}$. The differential geometry of wave fronts is discussed in [34]. Moreover, Martínez gave the following holomorphic representation for this class.

TheOrem 3.5. [27, Theorem 3] Let $\Sigma$ be a Riemann surface and $(F, G)$ a pair of holomorphic functions on $\Sigma$ such that $\operatorname{Re}(F d G)$ is exact and $|d F|^{2}+|d G|^{2}$ is positive definite. Then the induced map $\psi: \Sigma \rightarrow \mathbf{R}^{3}=\mathbf{C} \times \mathbf{R}$ given by

$$
\psi:=\left(G+\bar{F}, \frac{|G|^{2}-|F|^{2}}{2}+\operatorname{Re}\left(G F-2 \int F d G\right)\right)
$$

is an improper affine front. Conversely, any improper affine front is given in this way. Moreover we set $x:=G+\bar{F}$ and $n:=\bar{F}-G$. Then $L_{\psi}:=x+\sqrt{-1} n: \Sigma \rightarrow \mathbf{C}^{2}$ is a special Lagrangian immersion whose induced metric $d \tau^{2}$ from $\mathbf{C}^{2}$ is given by

$$
d \tau^{2}=2\left(|d F|^{2}+|d G|^{2}\right)
$$

In addition, the affine metric $h$ of $\psi$ is expressed as $h:=|d G|^{2}-|d F|^{2}$ and the singular points of $\psi$ correspond to the points where $|d F|=|d G|$.

The nontrivial part of the Gauss map of $L_{\psi}: \Sigma \rightarrow \mathbf{C}^{2} \simeq \mathbf{R}^{4}$ (see [6]) is the meromorphic function $\nu: \Sigma \rightarrow \mathbf{C} \cup\{\infty\}$ given by

$$
\nu:=\frac{d F}{d G}
$$

which is called the Lagrangian Gauss map of $\psi$. An improper affine front is said to be weakly complete if the induced metric $d \tau^{2}$ is complete. We note that

$$
d \tau^{2}=2\left(|d F|^{2}+|d G|^{2}\right)=2\left(1+|\nu|^{2}\right)|d G|^{2} .
$$

Applying Theorem 1.1 to the metric $d \tau^{2}$, we can get the following theorem. This is a generalization of [19, Theorem 3.2]. 
TheOREM 3.6. Let $\psi: \Sigma \rightarrow \mathbf{R}^{3}$ be an improper affine front whose Lagrangian Gauss map $\nu: \Sigma \rightarrow \mathbf{C} \cup\{\infty\}$ omits more than $3(=1+2)$ distinct values. Then there exists a positive constant $C$ depending on the set of exceptional values, but not $\Sigma$, such that for all $p \in \Sigma$ we have

$$
\left|K_{d \tau^{2}}(p)\right|^{1 / 2} \leq \frac{C}{d(p)},
$$

where $K_{d \tau^{2}}(p)$ is the Gaussian curvature of the metric $d \tau^{2}$ at $p$ and $d(p)$ is the geodesic distance from $p$ to the boundary of $\Sigma$. In particular, if the Lagrangian Gauss map of a weakly complete improper affine front in $\mathbf{R}^{3}$ is nonconstant, then it can omit at most 3 $(=1+2)$ values.

Since the singular points of $\psi$ correspond to the points where $|\nu|=1$, we can obtain a simple proof of the parametric affine Bernstein theorem ([3], [16]) for improper affine spheres from the viewpoint of value-distribution-theoretic properties of the Lagrangian Gauss map. For the proof, see [19, Corollary 3.6].

COROllary 3.7. Any affine complete improper affine sphere in $\mathbf{R}^{3}$ must be an elliptic paraboloid.

3.4. Ratio of canonical forms of flat fronts in the hyperbolic 3-space. We denote by $\mathbf{H}^{3}$ the hyperbolic 3-space, that is, the simply connected Riemannian 3-manifold with constant sectional curvature -1 , which is represented as

$$
\mathbf{H}^{3}=S L(2, \mathbf{C}) / S U(2)=\left\{a a^{*} ; a \in S L(2, \mathbf{C})\right\} \quad\left(a^{*}:={ }^{t} \bar{a}\right) .
$$

For a holomorphic Legendrian immersion $\mathcal{L}: \Sigma \rightarrow \mathbf{H}^{3}$ on a simply connected Riemann surface $\Sigma$, the projection

$$
f:=\mathcal{L L}^{*}: \Sigma \rightarrow \mathbf{H}^{3}
$$

gives a flat front in $\mathbf{H}^{3}$. Here, flat fronts in $\mathbf{H}^{3}$ are flat surfaces in $\mathbf{H}^{3}$ with some admissible singularities (see [23], [26] for the definition of flat fronts in $\mathbf{H}^{3}$ ). We call $\mathcal{L}$ the holomorphic lift of $f$. Since $\mathcal{L}$ is a holomorphic Legendrian map, $\mathcal{L}^{-1} d \mathcal{L}$ is off-diagonal (see [14], [25], $[26])$. If we set

$$
\mathcal{L}^{-1} d \mathcal{L}=\left(\begin{array}{cc}
0 & \theta \\
\omega & 0
\end{array}\right),
$$

then the pull-back of the canonical Hermitian metric of $S L(2, \mathbf{C})$ by $\mathcal{L}$ is represented as

$$
d s_{\mathcal{L}}^{2}:=|\omega|^{2}+|\theta|^{2}
$$

for holomorphic 1-forms $\omega$ and $\theta$ on $\Sigma$. A flat front $f$ is said to be weakly complete if the metric $d s_{\mathcal{L}}^{2}$ is complete $[24,37]$. We define a meromorphic function on $\Sigma$ by the ratio of canonical forms

$$
\rho:=\frac{\theta}{\omega}
$$


Then a point $p \in \Sigma$ is a singular point of $f$ if and only if $|\rho(p)|=1$ [22]. We note that

$$
d s_{\mathcal{L}}^{2}=|\omega|^{2}+|\theta|^{2}=\left(1+|\rho|^{2}\right)|\omega|^{2} .
$$

Applying Theorem 1.1 to the metric $d s_{\mathcal{L}}^{2}$, we can get the following theorem. This is a generalization of [19, Theorem 4.5].

THEOREM 3.8. Let $f: \Sigma \rightarrow \mathbf{H}^{3}$ be a flat front on a simply connected Riemann surface $\Sigma$. Suppose that the ratio of canonical forms $\rho: \Sigma \rightarrow \mathbf{C} \cup\{\infty\}$ omits more than $3(=1+2)$ distinct values. Then there exists a positive constant $C$ depending on the set of exceptional values, but not $\Sigma$, such that for all $p \in \Sigma$ we have

$$
\left|K_{d s_{\mathcal{L}}^{2}}(p)\right|^{1 / 2} \leq \frac{C}{d(p)},
$$

where $K_{d s_{\mathcal{L}}^{2}}(p)$ is the Gaussian curvature of the metric $d s_{\mathcal{L}}^{2}$ at $p$ and $d(p)$ is the geodesic distance from $p$ to the boundary of $\Sigma$. In particular, if the ratio of canonical forms of a weakly complete flat front in $\mathbf{H}^{3}$ is nonconstant, then it can omit at most $3(=1+2)$ values.

If $\Sigma$ is not simply connected, then we consider that $\rho$ is a meromorphic function on its universal covering surface $\widetilde{\Sigma}$. As a corollary of Theorem 3.8, we give a simple proof of the classification ([35], [39]) of complete nonsingular flat surfaces in $\mathbf{H}^{3}$. For the proof, see [17, Corollary 3.5].

COROLlary 3.9. Any complete nonsingular flat surface in $\mathbf{H}^{3}$ must be a horosphere or a hyperbolic cylinder.

\section{REFERENCES}

[1] L. Ahlfors, Zur Theorie der Überlagerungsflachen, Acta Math. 65 (1935), 157-194.

[2] L. J. Alías and B. Palmer, On the Gaussian curvature of maximal surfaces and the CalabiBernstein theorem, Bull. London Math. Soc. 33 (2001), 454-458.

[3] E. CALABI, Improper affine hypersurfaces of convex type and a generalization of a theorem by K. Jörgens, Mich. Math. J. 5 (1958), 108-126.

[4] E. CAlabi, Examples of Bernstein problems for some nonlinear equations, Proc. Sympos. Pure Math. 15 (1970), 223-230.

[5] S. S. Chern, Complex analytic mappings of Riemann surfaces I., Amer. J. Math. 82 (1960), 323337.

[6] B. Y. Chen and J. M. Morvan, Géométrie des surfaces lagrangiennes de $\mathbf{C}^{2}$, J. Math. Pures Appl. 66 (1987), 321-335.

[7] S. Y. Cheng and S. T. YaU, Maximal spacelike hypersurfaces in the Lorentz-Minkowski spaces, Ann. of Math. 104 (1976), 407-419.

[8] F. J. M. Estudillo And A. Romero, On maximal surfaces in the $n$-dimensional LorentzMinkowski space, Geom. Dedicate 38 (1991), 167-174.

[9] F. J. M. Estudillo And A. Romero, Generalized maximal surfaces in Lorentz-Minkowski space $L^{3}$, Math. Proc. Cambridge Philos. Soc. 111 (1992), 515-524. 
[10] F. J. M. Estudillo And A. Romero, On the Gauss curvature of maximal surfaces in the 3dimensional Lorentz-Minkowski space, Comment. Math. Helv. 69 (1994), 1-4.

[11] H. Fujimoto, On the number of exceptional values of the Gauss map of minimal surfaces, J. Math. Soc. Japan 40 (1988), 235-247.

[12] H. Fujimoto, Value Distribution Theory of the Gauss Map of Minimal Surfaces in $\mathbf{R}^{m}$, Aspects of Mathematics, E21. Friedr. Vieweg \& Sohn, Braunschweig, 1993.

[13] H. Fujimoto, Nevanlinna theory and minimal surfaces, Geometry V, 95-151, 267-272, Encyclopaedia Math. Sci., 90, Springer, Berlin, 1997.

[14] J. A. Gálvez, A. Martínez and F. Milán, Flat surfaces in hyperbolic 3-space, Math. Ann. 316 (2000), 419-435.

[15] T. Imaizumi And S. Kato, Flux of simple ends of maximal surfaces in $\mathbf{R}^{2,1}$, Hokkaido Math. J. 37 (2008), 561-610.

[16] K. Jörgens, Über die Lösungen der differentialgleichung $r t-s^{2}=1$, Math. Ann. 127 (1954), $130-134$.

[17] Y. KaWAKAmi, A ramification theorem for the ratio of canonical forms of flat surfaces in hyperbolic three-space, preprint, arXiv:1110.3110.

[18] Y. Kawakami, R. Kobayashi and R. Miyaoka, The Gauss map of pseudo-algebraic minimal surfaces, Forum Math. 20 (2008), 1055-1069.

[19] Y. KaWAKami And D. NAKAJo, Value distribution of the Gauss map of improper affine spheres, to appear in Journal of the Mathematical Society of Japan, arXiv:1004.1484.

[20] O. Kobayashi, Maximal surfaces in the 3-dimensional Minkowski space $\mathbf{L}^{3}$, Tokyo J. Math. 6 (1983), 297-309.

[21] R. KobAyAshi, Toward Nevanlinna theory as a geometric model for Diophantine approximation, Sugaku Expositions 16 (2003), 39-79.

[22] M. Kokubu, W. Rossman, K. Saji, M. Umehara and K. Yamada, Singularities of flat fronts in hyperbolic space, Pacific J. Math. 221 (2005), 303-351.

[23] M. Kokubu, W. Rossman, M. Umehara and K. Yamada, Flat fronts in hyperbolic 3-space and their caustics, J. Math. Soc. Japan 59 (2007), 265-299.

[24] M. Kokubu, W. Rossman, M. Umehara and K. Yamada, Asymptotic behavior of flat surfaces in hyperbolic 3-space, J. Math. Soc. Japan 61 (2009), 799-852.

[25] M. Kokubu, M. Umehara and K. Yamada, An elementary proof of Small's formula for null curves in PSL(2, C) and an analogue for Legendrian curves in PSL(2, C), Osaka J. Math. 40 (2003), 697-715.

[26] M. Kokubu, M. Umehara and K. Yamada, Flat fronts in hyperbolic 3-space, Pacific J. Math. 216 (2004), 149-175.

[27] A. Martínez, Improper affine maps, Math. Z. 249 (2005), 755-766.

[28] D. NAkAjo, A representation formula for indefinite improper affine spheres, Results Math. 55 (2009), 139-159.

[29] J. Noguchi And T. Ochiai, Geometric Function Theory in Several Complex Variables, Transl. Math. Monogr. 80, Amer. Math. Soc., Providence, RI, 1990.

[30] R. Osserman, Global properties of minimal surfaces in $E^{3}$ and $E^{n}$, Ann. of Math. 80 (1964), $340-364$.

[31] R. Osserman, A survey of minimal surfaces, second edition, Dover Publication Inc., New York, 1986. 
[32] A. Romero, Simple proof of Calabi-Bernstein's theorem on maximal surfaces, Proc. Amer. Math. Soc. 124 (1996), 1315-1317.

[33] M. Ru, Nevanlinna theory and its Relation to Diophantine Approximation, World Sci., River Edge, NJ, 2001.

[34] K. Saji, M. Umehara and K. Yamada, The geometry of fronts, Ann. of Math. 169 (2009), 491-529.

[35] S. SASAKI, On complete flat surfaces in hyperbolic 3-space, Kōdai Math Sem. Rep. 25 (1973), 449-457.

[36] M. Umehara and K. Yamada, Maximal surfaces with singularities in Minkowski space, Hokkaido Math. J. 35 (2006), 13-40.

[37] M. Umehara and K. Yamada, Applications of a completeness lemma in minimal surface theory to various classes of surfaces, Bull. London Math. Soc. 43 (2011), 191-199.

[38] M. Umehara and K. Yamada, CORRIGENDUM: Applications of a completeness lemma in minimal surface theory to various classes of surfaces, to appear in Bulletin of the London Mathematical Society. (doi:10.1112/blms/bds017)

[39] Y. A. Volkov and S. M. Vladimirova, Isometric immersions of the Euclidean plane in Lobačevskii space (Russian), Mat. Zametki 10 (1971), 327-332.

Graduate School of Science And Engineering,

YAMAGUCHI UNIVERSITY,

YAMAGUCHI, 753-8512, JAPAN

E-mail address: y-kwkami@yamaguchi-u.ac.jp 


\section{List of MI Preprint Series, Kyushu University}

The Global COE Program

Math-for-Industry Education \& Research Hub

MI

MI2008-1 Takahiro ITO, Shuichi INOKUCHI \& Yoshihiro MIZOGUCHI

Abstract collision systems simulated by cellular automata

MI2008-2 Eiji ONODERA

The intial value problem for a third-order dispersive flow into compact almost

Hermitian manifolds

MI2008-3 Hiroaki KIDO

On isosceles sets in the 4-dimensional Euclidean space

MI2008-4 Hirofumi NOTSU

Numerical computations of cavity flow problems by a pressure stabilized characteristiccurve finite element scheme

MI2008-5 Yoshiyasu OZEKI

Torsion points of abelian varieties with values in nfinite extensions over a padic field

MI2008-6 Yoshiyuki TOMIYAMA

Lifting Galois representations over arbitrary number fields

MI2008-7 Takehiro HIROTSU \& Setsuo TANIGUCHI

The random walk model revisited

MI2008-8 Silvia GANDY, Masaaki KANNO, Hirokazu ANAI \& Kazuhiro YOKOYAMA Optimizing a particular real root of a polynomial by a special cylindrical algebraic decomposition

MI2008-9 Kazufumi KIMOTO, Sho MATSUMOTO \& Masato WAKAYAMA

Alpha-determinant cyclic modules and Jacobi polynomials 
MI2008-10 Sangyeol LEE \& Hiroki MASUDA

Jarque-Bera Normality Test for the Driving Lévy Process of a Discretely Observed Univariate SDE

MI2008-11 Hiroyuki CHIHARA \& Eiji ONODERA

A third order dispersive flow for closed curves into almost Hermitian manifolds

MI2008-12 Takehiko KINOSHITA, Kouji HASHIMOTO and Mitsuhiro T. NAKAO

On the $L^{2}$ a priori error estimates to the finite element solution of elliptic problems with singular adjoint operator

MI2008-13 Jacques FARAUT and Masato WAKAYAMA

Hermitian symmetric spaces of tube type and multivariate Meixner-Pollaczek polynomials

MI2008-14 Takashi NAKAMURA

Riemann zeta-values, Euler polynomials and the best constant of Sobolev inequality

MI2008-15 Takashi NAKAMURA

Some topics related to Hurwitz-Lerch zeta functions

MI2009-1 Yasuhide FUKUMOTO

Global time evolution of viscous vortex rings

MI2009-2 Hidetoshi MATSUI \& Sadanori KONISHI

Regularized functional regression modeling for functional response and predictors

MI2009-3 Hidetoshi MATSUI \& Sadanori KONISHI

Variable selection for functional regression model via the $L_{1}$ regularization

MI2009-4 Shuichi KAWANO \& Sadanori KONISHI

Nonlinear logistic discrimination via regularized Gaussian basis expansions

MI2009-5 Toshiro HIRANOUCHI \& Yuichiro TAGUCHII

Flat modules and Groebner bases over truncated discrete valuation rings 
MI2009-6 Kenji KAJIWARA \& Yasuhiro OHTA

Bilinearization and Casorati determinant solutions to non-autonomous $1+1$ dimensional discrete soliton equations

\section{MI2009-7 Yoshiyuki KAGEI}

Asymptotic behavior of solutions of the compressible Navier-Stokes equation around the plane Couette flow

MI2009-8 Shohei TATEISHI, Hidetoshi MATSUI \& Sadanori KONISHI

Nonlinear regression modeling via the lasso-type regularization

MI2009-9 Takeshi TAKAISHI \& Masato KIMURA

Phase field model for mode III crack growth in two dimensional elasticity

MI2009-10 Shingo SAITO

Generalisation of Mack's formula for claims reserving with arbitrary exponents for the variance assumption

MI2009-11 Kenji KAJIWARA, Masanobu KANEKO, Atsushi NOBE \& Teruhisa TSUDA Ultradiscretization of a solvable two-dimensional chaotic map associated with the Hesse cubic curve

\section{MI2009-12 Tetsu MASUDA}

Hypergeometric T -functions of the q-Painlevé system of type $E_{8}^{(1)}$

MI2009-13 Hidenao IWANE, Hitoshi YANAMI, Hirokazu ANAI \& Kazuhiro YOKOYAMA A Practical Implementation of a Symbolic-Numeric Cylindrical Algebraic Decomposition for Quantifier Elimination

MI2009-14 Yasunori MAEKAWA

On Gaussian decay estimates of solutions to some linear elliptic equations and its applications

MI2009-15 Yuya ISHIHARA \& Yoshiyuki KAGEI

Large time behavior of the semigroup on $L^{p}$ spaces associated with the linearized compressible Navier-Stokes equation in a cylindrical domain 
MI2009-16 Chikashi ARITA, Atsuo KUNIBA, Kazumitsu SAKAI \& Tsuyoshi SAWABE Spectrum in multi-species asymmetric simple exclusion process on a ring

MI2009-17 Masato WAKAYAMA \& Keitaro YAMAMOTO

Non-linear algebraic differential equations satisfied by certain family of elliptic functions

MI2009-18 Me Me NAING \& Yasuhide FUKUMOTO

Local Instability of an Elliptical Flow Subjected to a Coriolis Force

MI2009-19 Mitsunori KAYANO \& Sadanori KONISHI

Sparse functional principal component analysis via regularized basis expansions and its application

MI2009-20 Shuichi KAWANO \& Sadanori KONISHI

Semi-supervised logistic discrimination via regularized Gaussian basis expansions

MI2009-21 Hiroshi YOSHIDA, Yoshihiro MIWA \& Masanobu KANEKO

Elliptic curves and Fibonacci numbers arising from Lindenmayer system with symbolic computations

MI2009-22 Eiji ONODERA

A remark on the global existence of a third order dispersive flow into locally Hermitian symmetric spaces

MI2009-23 Stjepan LUGOMER \& Yasuhide FUKUMOTO

Generation of ribbons, helicoids and complex scherk surface in laser-matter Interactions

MI2009-24 Yu KAWAKAMI

Recent progress in value distribution of the hyperbolic Gauss map

MI2009-25 Takehiko KINOSHITA \& Mitsuhiro T. NAKAO

On very accurate enclosure of the optimal constant in the a priori error estimates for $H_{0}^{2}$-projection 
MI2009-26 Manabu YOSHIDA

Ramification of local fields and Fontaine's property (Pm)

MI2009-27 Yu KAWAKAMI

Value distribution of the hyperbolic Gauss maps for flat fronts in hyperbolic three-space

MI2009-28 Masahisa TABATA

Numerical simulation of fluid movement in an hourglass by an energy-stable finite element scheme

MI2009-29 Yoshiyuki KAGEI \& Yasunori MAEKAWA Asymptotic behaviors of solutions to evolution equations in the presence of translation and scaling invariance

MI2009-30 Yoshiyuki KAGEI \& Yasunori MAEKAWA

On asymptotic behaviors of solutions to parabolic systems modelling chemotaxis

MI2009-31 Masato WAKAYAMA \& Yoshinori YAMASAKI

Hecke's zeros and higher depth determinants

MI2009-32 Olivier PIRONNEAU \& Masahisa TABATA

Stability and convergence of a Galerkin-characteristics finite element scheme of lumped mass type

MI2009-33 Chikashi ARITA

Queueing process with excluded-volume effect

MI2009-34 Kenji KAJIWARA, Nobutaka NAKAZONO \& Teruhisa TSUDA

Projective reduction of the discrete Painlevé system of type $\left(A_{2}+A_{1}\right)^{(1)}$

MI2009-35 Yosuke MIZUYAMA, Takamasa SHINDE, Masahisa TABATA \& Daisuke TAGAMI Finite element computation for scattering problems of micro-hologram using DtN map 
MI2009-36 Reiichiro KAWAI \& Hiroki MASUDA

Exact simulation of finite variation tempered stable Ornstein-Uhlenbeck processes

MI2009-37 Hiroki MASUDA

On statistical aspects in calibrating a geometric skewed stable asset price model

MI2010-1 Hiroki MASUDA

Approximate self-weighted LAD estimation of discretely observed ergodic OrnsteinUhlenbeck processes

MI2010-2 Reiichiro KAWAI \& Hiroki MASUDA

Infinite variation tempered stable Ornstein-Uhlenbeck processes with discrete observations

MI2010-3 Kei HIROSE, Shuichi KAWANO, Daisuke MIIKE \& Sadanori KONISHI Hyper-parameter selection in Bayesian structural equation models

MI2010-4 Nobuyuki IKEDA \& Setsuo TANIGUCHI

The Itô-Nisio theorem, quadratic Wiener functionals, and 1-solitons

MI2010-5 Shohei TATEISHI \& Sadanori KONISHI

Nonlinear regression modeling and detecting change point via the relevance vector machine

MI2010-6 Shuichi KAWANO, Toshihiro MISUMI \& Sadanori KONISHI

Semi-supervised logistic discrimination via graph-based regularization

MI2010-7 Teruhisa TSUDA

UC hierarchy and monodromy preserving deformation

MI2010-8 Takahiro ITO

Abstract collision systems on groups

MI2010-9 Hiroshi YOSHIDA, Kinji KIMURA, Naoki YOSHIDA, Junko TANAKA \& Yoshihiro MIWA

An algebraic approach to underdetermined experiments 
MI2010-10 Kei HIROSE \& Sadanori KONISHI

Variable selection via the grouped weighted lasso for factor analysis models

MI2010-11 Katsusuke NABESHIMA \& Hiroshi YOSHIDA

Derivation of specific conditions with Comprehensive Groebner Systems

MI2010-12 Yoshiyuki KAGEI, Yu NAGAFUCHI \& Takeshi SUDOU

Decay estimates on solutions of the linearized compressible Navier-Stokes equation around a Poiseuille type flow

MI2010-13 Reiichiro KAWAI \& Hiroki MASUDA

On simulation of tempered stable random variates

MI2010-14 Yoshiyasu OZEKI

Non-existence of certain Galois representations with a uniform tame inertia weight

MI2010-15 Me Me NAING \& Yasuhide FUKUMOTO

Local Instability of a Rotating Flow Driven by Precession of Arbitrary Frequency

MI2010-16 Yu KAWAKAMI \& Daisuke NAKAJO

The value distribution of the Gauss map of improper affine spheres

MI2010-17 Kazunori YASUTAKE

On the classification of rank 2 almost Fano bundles on projective space

MI2010-18 Toshimitsu TAKAESU

Scaling limits for the system of semi-relativistic particles coupled to a scalar bose field

MI2010-19 Reiichiro KAWAI \& Hiroki MASUDA

Local asymptotic normality for normal inverse Gaussian Lévy processes with high-frequency sampling

MI2010-20 Yasuhide FUKUMOTO, Makoto HIROTA \& Youichi MIE

Lagrangian approach to weakly nonlinear stability of an elliptical flow 
MI2010-21 Hiroki MASUDA

Approximate quadratic estimating function for discretely observed Lévy driven SDEs with application to a noise normality test

MI2010-22 Toshimitsu TAKAESU

A Generalized Scaling Limit and its Application to the Semi-Relativistic Particles System Coupled to a Bose Field with Removing Ultraviolet Cutoffs

MI2010-23 Takahiro ITO, Mitsuhiko FUJIO, Shuichi INOKUCHI \& Yoshihiro MIZOGUCHI Composition, union and division of cellular automata on groups

MI2010-24 Toshimitsu TAKAESU

A Hardy's Uncertainty Principle Lemma in Weak Commutation Relations of Heisenberg-Lie Algebra

MI2010-25 Toshimitsu TAKAESU

On the Essential Self-Adjointness of Anti-Commutative Operators

MI2010-26 Reiichiro KAWAI \& Hiroki MASUDA

On the local asymptotic behavior of the likelihood function for Meixner Lévy processes under high-frequency sampling

MI2010-27 Chikashi ARITA \& Daichi YANAGISAWA

Exclusive Queueing Process with Discrete Time

MI2010-28 Jun-ichi INOGUCHI, Kenji KAJIWARA, Nozomu MATSUURA \& Yasuhiro OHTA

Motion and Bäcklund transformations of discrete plane curves

MI2010-29 Takanori YASUDA, Masaya YASUDA, Takeshi SHIMOYAMA \& Jun KOGURE On the Number of the Pairing-friendly Curves

MI2010-30 Chikashi ARITA \& Kohei MOTEGI

Spin-spin correlation functions of the $q$-VBS state of an integer spin model

MI2010-31 Shohei TATEISHI \& Sadanori KONISHI

Nonlinear regression modeling and spike detection via Gaussian basis expansions 
MI2010-32 Nobutaka NAKAZONO

Hypergeometric $\tau$ functions of the $q$-Painlevé systems of type $\left(A_{2}+A_{1}\right)^{(1)}$

MI2010-33 Yoshiyuki KAGEI

Global existence of solutions to the compressible Navier-Stokes equation around parallel flows

MI2010-34 Nobushige KUROKAWA, Masato WAKAYAMA \& Yoshinori YAMASAKI

Milnor-Selberg zeta functions and zeta regularizations

MI2010-35 Kissani PERERA \& Yoshihiro MIZOGUCHI

Laplacian energy of directed graphs and minimizing maximum outdegree algorithms

MI2010-36 Takanori YASUDA

CAP representations of inner forms of $S p(4)$ with respect to Klingen parabolic subgroup

MI2010-37 Chikashi ARITA \& Andreas SCHADSCHNEIDER

Dynamical analysis of the exclusive queueing process

MI2011-1 Yasuhide FUKUMOTO\& Alexander B. SAMOKHIN

Singular electromagnetic modes in an anisotropic medium

MI2011-2 Hiroki KONDO, Shingo SAITO \& Setsuo TANIGUCHI

Asymptotic tail dependence of the normal copula

MI2011-3 Takehiro HIROTSU, Hiroki KONDO, Shingo SAITO, Takuya SATO, Tatsushi TANAKA \& Setsuo TANIGUCHI

Anderson-Darling test and the Malliavin calculus

MI2011-4 Hiroshi INOUE, Shohei TATEISHI \& Sadanori KONISHI

Nonlinear regression modeling via Compressed Sensing

MI2011-5 Hiroshi INOUE

Implications in Compressed Sensing and the Restricted Isometry Property

MI2011-6 Daeju KIM \& Sadanori KONISHI

Predictive information criterion for nonlinear regression model based on basis expansion methods

MI2011-7 Shohei TATEISHI, Chiaki KINJYO \& Sadanori KONISHI

Group variable selection via relevance vector machine 
MI2011-8 Jan BREZINA \& Yoshiyuki KAGEI

Decay properties of solutions to the linearized compressible Navier-Stokes equation around time-periodic parallel flow

Group variable selection via relevance vector machine

MI2011-9 Chikashi ARITA, Arvind AYYER, Kirone MALLICK \& Sylvain PROLHAC Recursive structures in the multispecies TASEP

MI2011-10 Kazunori YASUTAKE

On projective space bundle with nef normalized tautological line bundle

MI2011-11 Hisashi ANDO, Mike HAY, Kenji KAJIWARA \& Tetsu MASUDA

An explicit formula for the discrete power function associated with circle patterns of Schramm type

MI2011-12 Yoshiyuki KAGEI

Asymptotic behavior of solutions to the compressible Navier-Stokes equation around a parallel flow

MI2011-13 Vladimír CHALUPECKÝ \& Adrian MUNTEAN

Semi-discrete finite difference multiscale scheme for a concrete corrosion model: approximation estimates and convergence

MI2011-14 Jun-ichi INOGUCHI, Kenji KAJIWARA, Nozomu MATSUURA \& Yasuhiro OHTA

Explicit solutions to the semi-discrete modified KdV equation and motion of discrete plane curves

MI2011-15 Hiroshi INOUE

A generalization of restricted isometry property and applications to compressed sensing

MI2011-16 Yu KAWAKAMI

A ramification theorem for the ratio of canonical forms of flat surfaces in hyperbolic three-space

MI2011-17 Naoyuki KAMIYAMA

Matroid intersection with priority constraints

MI2012-1 Kazufumi KIMOTO \& Masato WAKAYAMA

Spectrum of non-commutative harmonic oscillators and residual modular forms

MI2012-2 Hiroki MASUDA

Mighty convergence of the Gaussian quasi-likelihood random fields for ergodic

Levy driven SDE observed at high frequency

MI2012-3 Hiroshi INOUE

A Weak RIP of theory of compressed sensing and LASSO 
MI2012-4 Yasuhide FUKUMOTO \& Youich MIE

Hamiltonian bifurcation theory for a rotating flow subject to elliptic straining field

MI2012-5 Yu KAWAKAMI

On the maximal number of exceptional values of Gauss maps for various classes of surfaces 